\title{
Evidenzbasierte Praxis in der frühen Kindheit
}

\author{
Kathrin Keller-Schuhmacher
}

Das Konzept der Evidenzbasierten Praxis in der Medizin hat zunehmend Eingang in andere Bereiche gefunden, in denen es unterschiedlich rezipiert und teilweise kontrovers diskutiert wird. Demgegenüber stellen Buysse \& Wesley 2006 in "Evidence-Based Practice in the Early Childhood Field" eine Definition von Evidenzbasierter Praxis (EBP) im Handlungsfeld der frühen Kindheit vor, die dessen Besonderheiten Rechnung trägt und auf die Förderung des Dialogs zwischen Wissenschaft und Praxis abzielt. Die Autorinnen bieten eine handlungsleitende Definition von EBP an und zeigen ein konkretes Vorgehen auf, wie Fachpersonen aus den verschiedenen Disziplinen und Berufsfeldern in einen Dialog über eine zeitgemäße Entwicklungsbegleitung von Kindern und ihren Familien treten können.

\section{Evidenzbasierte Praxis in der frühen Kindheit - offene Fragen}

Beim Blick in die Literatur zu EBP im Allgemeinen und zur Übertragung des Konzeptes aus der Medizin ${ }^{1}$ in andere Felder fällt Folgendes auf:

1. Es besteht Uneinigkeit darüber, was als evident zählt: „Auf der einen Seite (...) stehen jene, die ausschließlich die Resultate von Studien mit einem randomisierten Kontrollgruppendesign (...) akzeptieren. Die andere Seite des Extrems bilden jene, die persönliche Erfahrung oder die Meinung von Fachexperten als Quellen für Evidenz be-

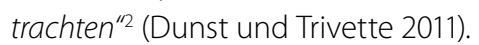

2. Es wird in Frage gestellt, ob sich das Konzept auf andere Bereiche, wie Pädagogik und Bildungsforschung, übertragen lässt (z. B. Jornitz 2009, Forster 2014)
3. In den USA liegt der Schwerpunkt in der frühen Kindheit auf Entwicklungsangemessener Praxis, definiert als „... ein Rahmen, der ideale erzieherische Praktiken zusammenfasst, die kindliches Lernen und kindliche Entwicklung mittels eines Prozesses fördern, in dem Ziele identifiziert und bewusste Entscheidungen getroffen werden, die die Erreichung dieser Ziele erleichtern". Bei dieser Entscheidungsfindung seien die allgemeine Entwicklung und die Art des Lernens des individuellen Kindes und dessen soziale und kulturelle Werte und Erwartungen zu beachten (NAECY, in Farley et al. 2018, 2). Im Handlungsfeld frühe Kindheit wird oft nicht unterschiedenzwischen „Entwicklungsangemessener Praxis" und "Evidenzbasierter Praxis" (Farley et al. 2018).

4. "Early Childhood”, Geburt bis 8 Jahre in den USA $^{3}$, wird im deutschsprachigen Raum definiert als "Frühe Kindheit" 0 bis $3^{4}$ bzw. bis 6 Jahre $^{5}$.

\section{Evidenzbasierte Praxis in der frühen Kindheit - eine Klärung}

Ausgangspunkt für die Auseinandersetzung mit EBP in der frühen Kindheit war die Frage von Buysse und Wesley, wie sich die Lücke zwischen Forschung und Praxis in der frühen Kindheit schließen ließe, wenn alle Beteiligten und Interessierten aus Praxis und Wissenschaft - Fachpersonen aus der Praxis, Eltern, Forscher und andere, die auf der Suche nach Wissen und dessen Umsetzung in die Praxis sind - in Communities of Practice zusammengebracht werden könnten. Auf dieser Lern- und Entdeckungsreise sind sie auf die „Bewegung" der EBP gestoßen. Sie stellten 
fest, dass diese zunehmend einen bedeutenden Einfluss auf viele verschiedene Disziplinen und Berufe ausübte und dass sie das Potenzial haben könnte, die Art und Weise, wie Wissen im Feld der frühen Kindheit generiert, verbreitet und verwendet wird, entscheidend zu verändern.

Ihr Anliegen war es, eine Definition von EBP in der frühen Kindheit zu finden, die direkte Auswirkungen auf das Handeln der Fachpersonen in der Praxis hat. Es war innen bewusst, .... dass die Arena der Frühen Kindheit nicht länger auf der Grundlage von Ideologie und kollektiver Weisheit vorwärtskommen kann, sondern sich durch bedachte Integration laufender wissenschaftlicher Untersuchungen informieren muss"(Shonkoff, in Buysse und Wesley 2006, xi).

In Erweiterung der Definition von EBP in der Medizin umschreiben Buysse und Wesley Evidenzbasierte Praxis in der frühen Kindheit „... als einen Entscheidungsprozess, bei dem die beste verfügbare Forschungsevidenz mit den Werten und dem Wissen von Familien und Fachpersonen integriert werden" (Buysse und Wesley 2006, xiv).

Evidenzbasierte Praxis in der frühen Kindheit wird demnach durch externe, interne und soziale Evidenz charakterisiert. ${ }^{6}$ In den von mir ausgewählten Publikationen aus dem deutschsprachigen Raum habe ich denn auch dieses Verständnis von EBP gefunden (z. B. Burgener Woeffray et al. 2016, Pretis 2016).

Gemäß Buysse und Wesley spiegelt diese Definition eine tiefgreifende Veränderung gegenüber einem Denken wider, das davon ausgeht, dass sich Praxiswissen verändern lässt, indem der Praxis geschriebene Anleitungen für Methoden vorgelegt werden, die von Expertengruppen oder professionellen Organisationen ausgearbeitet und genehmigt worden waren. Obige Definition von EBP in der frühen Kindheit geht von einer Demokratisierung des Wissens aus: Dieses wird transparent und für alle verfügbar. Das bedingt, dass Fachpersonen in der Praxis die Evidenz identifizieren, evaluieren, interpretieren und sie zur Lösung praktischer Probleme anwenden können.
Als Herzstück dieser Definition bezeichnen die Autorinnen denn auch, dass EBP in der frühen Kindheit als ein Prozess - ein Verfahren - verstanden wird, bei dem sich Fachpersonen in der frühen Kindheit ${ }^{7}$ befähigen, im Interesse von Kindern und Familien wirksam zu handeln.

Professionelles Wissen sollte so organisiert werden können, dass es

1. Antworten auf unmittelbare Fragen aus der Praxis gibt (Relevanz),

2. den Blick auf die Verbesserung von Ergebnissen richtet (Wichtigkeit),

3. verschiedene Quellen von Wissen integriert (Wirksamkeit),

4. breit verfügbar und zugänglich ist (Nützlichkeit) (Buysse 2012).

Ein Weg zu solcher Wissensorganisation in der Praxis öffnet sich über Communities of Practice:

"In Early Intervention and Education one approach to integrate theory and practice is to create communities of practice, that, as in the above description are based in collective expertise and designed to stimulate a bidirectional flow of knowledge from research to practice and from practice to research." (Buysse und Wesley 2006, 163)

Unter diesem Aspekt verstehe ich die Definition von EBP in der frühen Kindheit sensu Buysse und Wesley auch als einen "Aufruf", die Praxis zu ermutigen und zu stärken, sich an der Generierung, Identifikation und Verbreitung von Wissen zu beteiligen. Nicht zuletzt auch, indem traditionelle Vorstellungen über Lernen als Aneignung von Wissen zugunsten einer neueren Sicht auf Lernen durch Partizipation ${ }^{9}$ abgelöst werden (Buysse und Wesley 2006).

\section{. last but not least}

Evidenzbasierte Interventionen in der frühen Kindheit grundsätzlich zu überdenken, dazu fordert Shonkoff 2017 auf. Er stellt fest, dass das be- 
grenzte Ausmaß, in dem neue Erkenntnisse aus Neurowissenschaften, Molekularbiologie und Epigenetik genutzt werden, um die sozialen Determinanten von Entwicklung anzugehen, nicht länger vertretbar sei, da eine gesunde Hirnentwicklung Schutz vor übermäßigem Stress und nicht lediglich mehr Anregung in einer stimulierenden Umgebung erfordere: „Neue Einsichten über Neuroplastizität und kritische Perioden in der Entwicklung könnten neues Denken über das Timing und die Art von Interventionen über die Lebensspanne anregen. Valide Messungen von biologischen und verhaltenswirksamen Auswirkungen von erheblichem Stress bei kleinen Kindern, (...) könnten wegweisende Implikationen haben." (Shonkoff 2017, 1)

Herzlichen Dank an Doris Grauwiler und Michael Watson für ihre hilfreichen Kommentare.

\section{Anmerkungen}

1 Sackett et al. 1996 umschreiben Evidenzbasierte Praxis in der Medizin als „... die gewissenhafte, explizite und vernünftige Anwendung der besten aktuellen Evidenz bei der Entscheidung über die Behandlung individueller Patienten. (...) Es bedeutet, individuelle klinische Expertise und die beste externe klinische Evidenz aus der systematischen Forschung zu integrieren." (Sackett et al. 1996, 71)

2 Alle Übersetzungen aus dem Amerikanischen: Kathrin Keller-Schuhmacher

3 NAECY = National Association for the Education of Young Children: https://www.naeyc.org/);

4 Gesellschaft für seelische Gesundheit in der frühen Kindheit, www.gaimh.org

5 Deutsche Liga für das Kind, www.liga-kind.de

6 vgl. auch Dollaghan 2007 (in Kuhr und Kuwaliak 2018), die diese drei Quellen von Evidenz für die Sprachbehindertenpädagogik ausgemacht hatte. Hingegen wird in der Frühpädagogik in Großbritannien lediglich externe Evidenz als Quelle definiert (Lloyd 2015).

7 In diesem Feld sind die verschiedensten Disziplinen und Berufe vertreten, „... early childhood education, early intervention, child care, infant and child mental health, developmental and clinical psychology, social work, the medical and allied health professions among others"(Buysse und Wesley 2006, xiiif). Analog sehe ich die Zusammensetzung des Handlungsfeldes „Frühe Kindheit" in den deutschsprachigen Ländern. Demgegenüber bezeichnen Farley et al. 2018 jene Fachpersonen als "Early Childhood Practitioners", die in "Early Education" und "Special Early Education"tätig sind. Das betrifft jene Fachpersonen, die in Kita und Spielgruppe (CH: Frühe Förderung/Frühkindliche Bildung, Betreuung und Erziehung) oder in der Frühförderung ( $\mathrm{CH}$ : Heilpädagogische Früherziehung) arbeiten. Farley et al. 2018 stützen sich bei der Definition von "Evidence-based Practice in Early Childhood Education" auf jene des "Every Student Succeeds Act (ESSA)". Diese umschreibt EBP als „... eine Aktivität, Strategie oder Intervention, die die Ergebnisse des Schülers statistisch signifikant verbessern", wobei die Wirkung mindestens in einer strengen experimentellen, quasi-experimentellen oder Korrelationsstudie nachgewiesen sein muss (Farley et al. 2018, 2). Diese Definition stammt aus dem Bereich des Schulunterrichts, der nach meinem Verständnis der (Selbst-)Bildungs- und Lernprozesse in der frühen Kindheit nichts zu suchen hat.

8 Das Konzept der Communities of practice wurde 1998 von Wenger entwickelt (vgl. auch Wenger 2000 und Wenger 2010). Es stellt Lernen in den Kontext sozialer Beziehungen. Bliss et al. 2006 beschreiben, wie in Communities of Practice die Wissensgenerierung vergemeinschaftet wird.

9 Zu „Bildung durch Partizipation“ vgl. auch das eben erschienene Buch von Gerd E. Schäfer (2019): Bildung durch Beteiligung - Zur Praxis und Theorie frühkindlicher Bildung. Beltz, Weinheim.

\section{Literatur}

Bliss, F. R., Johanning, A., Schicke, H. (2006): Communities of Practice - Ein Zugang zu sozialer Wissensgenerierung. Deutsches Institut für Erwachsenenbildung. In: http://www.die-bonn.de/esprid/doku mente/doc-2006/bliss06_01.pdf, 2.9.2019

Burgener Woeffray, A., Lanfranchi, A., Koch, C. (2016): Wirksamkeit an Evidenzen Messen. Schweizerische Zeitschrift für Heilpädagogik Jg. 22, 3/2016

Buysse, V., Wesley, P.W. (Hrsg.) (2006): Evidence Based Practice in the Early Childhood Field. ZERO TO THREE, Washington DC, https://doi.org/10.1177/109 625060600900401

Buysse, V. (2012): Evidenz-Based Practice: What this Means for Practitioners? Referat anlässlich GAIMH Tagung 2012 in Basel. In: https://www.gaimh.org/ jahrestagung-basel-2012.html, 2.9.2019 
Farley, K. S., Brock, M. E., Winterbottom, C. (2018): Evidence-Based Practice: Providing Guidance for Early Childhood Practitioners. Journal of Research in Childhood Education 32 (1), 1 - 13, https://doi.org/10.1080/ 02568543.2017 .1387205

Dunst, C. J., Trivette, C. M. (2011): Evidence-Based Practices in Early Childhood Intervention and Family Support. Presentation made at the Celebration Lecture Series, Center for Excellence in Early Childhood Learning and Development, East Tennessee State University, Johnson City, February 4, 2011. In: http:// www.puckett.org/presentations/EvidBasedPracEarly Childhood_2_2011.pdf, 4.6.2019

Forster, E. (2014): Kritik der Evidenz: Das Beispiel evidence-informed policy research der OECD. Zeitschrift für Pädagogik 60 (2014) 6, 890-907. URN: urn:nbn:de:0111-pedocs-1 46880

Jornitz, S. (2009): Evidenzbasierte Bildungsforschung. Pädagogische Korrespondenz (2009) 40, 68-75, URN: urn:nbn:de:0111-opus-57256

Kuhr, L., Kulawiak, P. R. (2018): Wissen, was wirkt: Modelle evidenzbasierter Pädagogik. Potsdamer Zentrum für empirische Inklusionsforschung, 2018, Nr. 07

Lloyd, E. (2015): Evidenzbasierte Praxis und Politik in der Frühpädagogik in Großbritannien. In: Sterdt, E., Schmitt, A., Morfeld, M., Fischer, L. (Hrsg): Evidenz- basierte Praxis und Politik in der Frühpädagogik. Ein Tagungsbericht. Halle, Mitteldeutscher Verlag, https://doi.org/10.1055/s-0035-1563292

Pretis, M. (2016): ICF-basiertes Arbeiten in der Frühförderung. Ernst Reinhardt, München

Sackett, D. L., Rosenberg, W. M., Gray, J. A., Haynes, R. B., Richardson, W. S. (1996): Evidence based medicine: what it is and what it isn't. British Medical Journal, Jan 13; 312 (7023), 71 - 72, https://doi.org/ 10.1136/bmj.312.7023.71

Shonkoff, J.P. (2006): Vorwort zu Buysse, V., Wesley, P.W. (Hrsg.): Evidence Based Practice in the Early Childhood Field. Washington DC, ZERO TO THREE Shonkoff, J. P. (2017): Rethinking the Definition of Evidence-Based Interventions to Promote Early Childhood Development. PEDIATRICS 140 (6), December 2017: e20173136, https://doi.org/10.1542/ peds.2017-3136, 14.6. 2019

Wenger, E. (2000): Communities of Practice and Social Learning Systems. Organization 7 (2), 225 -246, https://doi.org/10.1177/135050840072002

Wenger, E. (2010): Communities of practice and social learning systems: the career of a concept. In: Blackmore, C. (Hrsg.) (2010): Social Learning Systems and Communities of Practice. London, Springer, https://doi.org/10.1007/978-1-84996-133-2_11 